\title{
High temperature superconductivity in sulfur and selenium hydrides at high pressure ${ }^{\star}$
}

\author{
José A. Flores-Livas, Antonio Sanna ${ }^{\mathrm{a}}$, and E.K.U. Gross \\ Max-Planck Institut für Microstrukture Physics, Weinberg 2, 06120 Halle, Germany
}

Received 12 January 2016

Published online 7 March 2016

(C) The Author(s) 2016. This article is published with open access at Springerlink.com

\begin{abstract}
Due to its low atomic mass, hydrogen is the most promising element to search for hightemperature phononic superconductors. However, metallic phases of hydrogen are only expected at extreme pressures (400 $\mathrm{GPa}$ or higher). The measurement of the record superconducting critical temperature of $203 \mathrm{~K}$ in a hydrogen-sulfur compound at $160 \mathrm{GPa}$ of pressure [A.P. Drozdov, M.I. Eremets, I.A. Troyan, arXiv:1412.0460 [cond-mat.supr-con] (2014); A.P. Drozdov, M.I. Eremets, I.A. Troyan, V. Ksenofontov, S.I. Shylin, Nature 525, 73 (2015)], shows that metallization of hydrogen can be reached at significantly lower pressure by inserting it in the matrix of other elements. In this work we investigate the phase diagram and the superconducting properties of the H-S systems by means of minima hopping method for structure prediction and density functional theory for superconductors. We also show that Se-H has a similar phase diagram as its sulfur counterpart as well as high superconducting critical temperature. We predict $\mathrm{H}_{3}$ Se to exceed $120 \mathrm{~K}$ superconductivity at $100 \mathrm{GPa}$. We show that both $\mathrm{H}_{3} \mathrm{Se}$ and $\mathrm{H}_{3} \mathrm{~S}$, due to the critical temperature and peculiar electronic structure, present rather unusual superconducting properties.
\end{abstract}

Under high pressure conditions, insulating and semiconducting materials tend to become metallic, because, with increasing electronic density, the kinetic energy grows faster than the potential energy. As metallicity is a necessary condition for superconductivity, generally becomes more likely under pressure [1,2]. Wigner and Huntington [3], already in 1935 suggested the possibility of a metallic modification of hydrogen under very high pressures. Ashcroft and Richardson predicted [4,5] hydrogen to become metallic under pressure and also the possibility to be a high temperature superconductor. The high critical temperature $\left(T_{\mathrm{C}}\right)$ of hydrogen $[6-8]$ is a consequence of its low atomic mass leading to high energy vibrational modes and in turn to a large phase space available for electronphonon scattering to induce superconductivity [9]. However, the estimated metallization pressure $[10,11]$ is beyond the current experimental capabilities [12-15]. It was only recently that hydrogen-rich compounds (chemically pre-compressed) started to be explored as a way to decrease the tremendous metallization pressure of pure hydrogen [16]. The first system explored experimentally was silane $\left(\mathrm{SiH}_{4}\right)$ [17]. Soon after, many others materials have been explored experimentally [18-21] and theoretically [22-40].

\footnotetext{
* Supplementary material in the form of one pdf file available from the Journal web page at:

http://dx.doi.org/10.1140/epjb/e2016-70020-0

a e-mail: sanna@mpi-halle.mpg.de
}

Recent resistivity and magnetic measurements on sulfur hydride $\left(\mathrm{H}_{2} \mathrm{~S}\right)$, demonstrate that when pressurized, becomes metallic and superconducting. For pressures above $160 \mathrm{GPa}$, a record breaking transition temperature of $200 \mathrm{~K}$ was confirmed [41,42]. This $T_{\mathrm{C}}$ is the highest measured in any superconductor known so far, including pnictides and cuprates $[43,44]$. And the experimental evidence was immediately supported by calculations $[32,35-37,39,45,46]$. In this work [47] we want study in detail the $\mathrm{S}-\mathrm{H}$ phases with state of the art ab-initio material search minima hopping method (MHM) [48-50] and compute the superconducting properties with the completely parameter-free Density Functional Theory for Superconductors (SCDFT) [51]. At the same time we also extend the analysis to the Se-H system, predicting a fairly similar phase diagram and comparable superconducting properties.

In order to predict the global minimum structure of sulfur/selenium hydride compounds we use MHM for the prediction of low-enthalpy structures. This method has been successfully used for global geometry optimization in a large variety of applications [40,52-54]. Given only the chemical composition of a system, MHM aims at finding the global minimum on the enthalpy surface while gradually exploring low-lying structures. Moves on the enthalpy surface are performed by using variable cell shape molecular dynamics with initial velocities approximately chosen along soft mode directions. We have used 1, 2, 3 

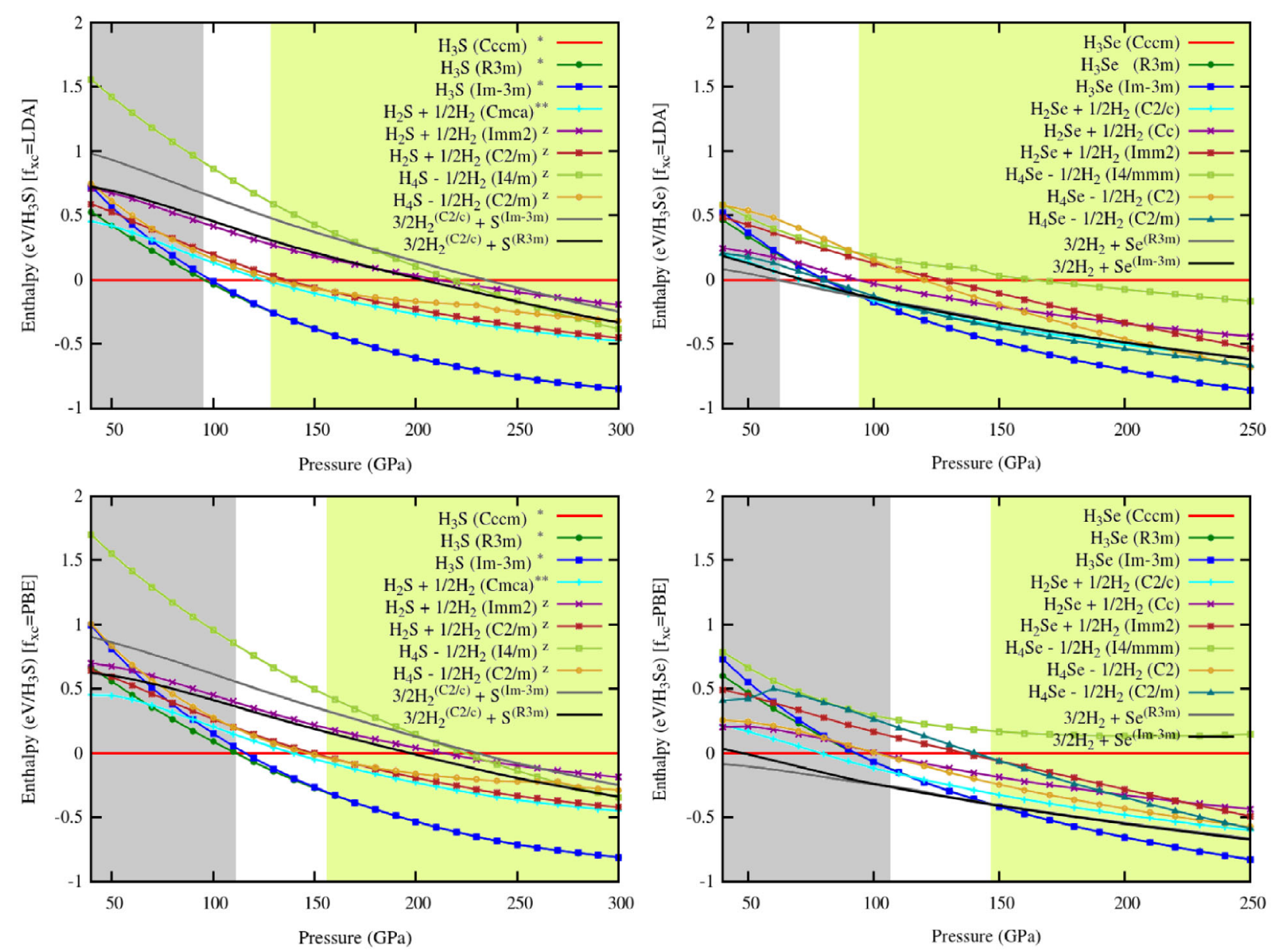

Fig. 1. Computed LDA and GGA-PBE enthalpy diagrams for $\mathrm{H}_{n} \mathrm{~S}$ and $\mathrm{H}_{n} \mathrm{Se}(n=2,3,4)$ as function of pressure. Values are given with respect to the $\mathrm{Cccm}$ structure, stable at low pressure. We found in our runs for $\mathrm{H}_{3} \mathrm{~S}$ three structures reported in reference [35] $\left(^{*}\right)$ and two in reference [32] (**). The other structures with compositions $\mathrm{H}_{2} \mathrm{~S}, \mathrm{H}_{4} \mathrm{~S}$ as well as all the structures of $\mathrm{H}_{2} \mathrm{Se}, \mathrm{H}_{3} \mathrm{Se}$ and $\mathrm{H}_{4} \mathrm{Se}$ are predictions of this work.

formula units of $\mathrm{H}_{n} \mathrm{~S}$ and $\mathrm{H}_{n} \mathrm{Se}(n=2,3,4)$ at selected pressures of 40, 80, 100, 150, 200, 250, 300 and $400 \mathrm{GPa}$. The relaxations to local minima are performed by the fast inertia relaxation engine [55] taking into account, both atomic and cell degrees of freedom. Final structural relaxations and enthalpy calculations were performed using the local density approximation LDA and GGA to the exchange correlation functional, as implemented in the VASP code [56]. The plane-wave cutoff energy was set to $800 \mathrm{eV}$, and $\Gamma$-center Monkhorst-Pack $k$-point meshes with grid spacing denser than $2 \pi \times 0.01 \AA^{-1}$, resulting in total energy convergence better than $2 \mathrm{meV} /$ atom. Electronic and phononic structure calculations were performed within DFT as implemented in the two plane-wave based codes ABINIT [57], and ESPRESSO [58] within the local density approximation LDA exchange correlation functional. Superconducting properties have been computed within density functional for superconductors (SCDFT) $[51,59,60]$, the method was described in previous references $[59,60]$. This theory of superconductivity is completely ab-initio, fully parameter-free and proved to be accurate and successful in describing phononic superconductors [61-65]. It allows to compute all superconducting properties includ- ing the critical temperature and the excitation spectrum of the system. Coulomb interactions are included within static RPA [63], therefore excluding magnetic source of coupling [66]. In this work we used an improved functional form (also used in Ref. [65]) that will be fully described elsewhere. The difference between this new functional and the one from Lueders and Marques is the inclusion of Migdal's theorem in a rigorous way like in Eliashberg theory. This is important because this ensures that the phononic description is accurate at least in the limit in which the electronic characteristic energy is large compared to the phononic scale. While the Lueders and Marques theory $[59,60]$ does not contain this exact limiting behaviour.

Experimentally little is known on the high pressure stability and composition of the $\mathrm{S}-\mathrm{H}$ system and, to the best of our knowledge, nothing is known about Se-H. Therefore we investigate the compositional and configurational space of $\mathrm{H}_{x} \mathrm{~S}$ and $\mathrm{H}_{x}$ Se with a sophisticated structure prediction method. Computed enthalpies as a function of pressure are reported in Figure 1. We consider the $\mathrm{H}_{3} \mathrm{~S}$ stoichiometry (left panels) as well as its elemental decomposition (sulfur + hydrogen), its decomposition into 

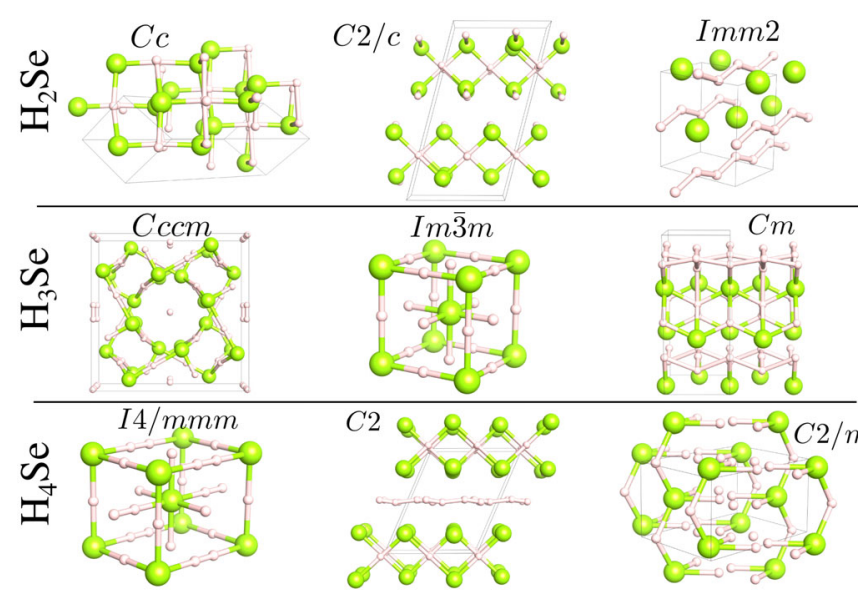

Fig. 2. Low-lying enthalpy structures found for different compositions at selected pressures. The large and small spheres denote the Se and $\mathrm{H}$ atoms, respectively.

$\mathrm{H}_{2} \mathrm{~S}+$ hydrogen and $\mathrm{H}_{4} \mathrm{~S}$-hydrogen. At low pressure we find the $C c c m$ structure up to $95 \mathrm{GPa}$ and the $R 3 m(\beta$-Potype) rhombohedral structure between 95 and $150 \mathrm{GPa}$. Above $150 \mathrm{GPa}$, we confirm the cubic $\operatorname{Im} \overline{3} m$ (bcc) as the most stable structure in perfect agreement with the experiments of Einaga et al. [67]. The results also indicate that for low pressures the 2:1 composition is stable, however as pressure increases at above $40 \mathrm{GPa}$ the $3: 1$ is the dominant stoichiometry and remains so up to $300 \mathrm{GPa}$. Another H-rich compound, 4:1 is unstable in the pressure range of this study and these results are in agreement with the work of Duan et al. [38]. In a similar way we have studied the Se-H phase diagram. Chemically, selenium is known to have very similar physical properties to sulfur and this system is not an exception. Enthalpies are shown in Figure 1 (right panels). Once again we use the Cccm structure as reference since, as in the case of sulfur, it is the most stable at low pressures and up to $80 \mathrm{GPa}$. Between $80 \mathrm{GPa}$ to $100 \mathrm{GPa}$, we find that the $\mathrm{H}_{2} \mathrm{Se}+$ hydrogen decomposition is more stable than the $\mathrm{H}_{3}$ Se stoichiometry. $\mathrm{H}_{3}$ Se turns out to be the most stable composition above $100 \mathrm{GPa}$ and at least up to $250 \mathrm{GPa}$. As in the case of sulfur-hydride, the 4:1 composition of $\mathrm{H}$ :Se is not thermodynamically stable in the phases predicted and region of pressure studied.

Therefore from our analysis both systems in the range $50 \mathrm{GPa}$ to $250 \mathrm{GPa}$ show, with increasing pressure, two phase transitions. The $\mathrm{S}-\mathrm{H}$ system, always stable in the $\mathrm{H}_{3} \mathrm{~S}$ stoichiometry, has a first order phase transition from $C c c m$ to $R 3 m$ at $\sim 100 \mathrm{GPa}$, then the $R 3 m$ rhombohedral distortion decreases continuously up to $150 \mathrm{GPa}$, pressure at which it transforms into the $\operatorname{Im} \overline{3} m$ cubic structure. The $\mathrm{H}_{3}$ Se cubic structure is depicted in Figure 2. The Se-H system at low pressure (LDA) is also stable in the $\mathrm{H}_{3}$ Se stoichiometry but becomes unstable to a phase separation into $\mathrm{H}_{2} \mathrm{Se}+$ hydrogen in the range from $70 \mathrm{GPa}$ to $100 \mathrm{GPa}$. Above $100 \mathrm{GPa}$ another discontinuous phase transition occurs, directly into the $\operatorname{Im} \overline{3} m$ cubic structure. Note that $100 \mathrm{GPa}$ is also the pressure below in which the $\operatorname{Im} \overline{3} m$ structure would distort into the $R 3 m$ ( $\beta$-Po

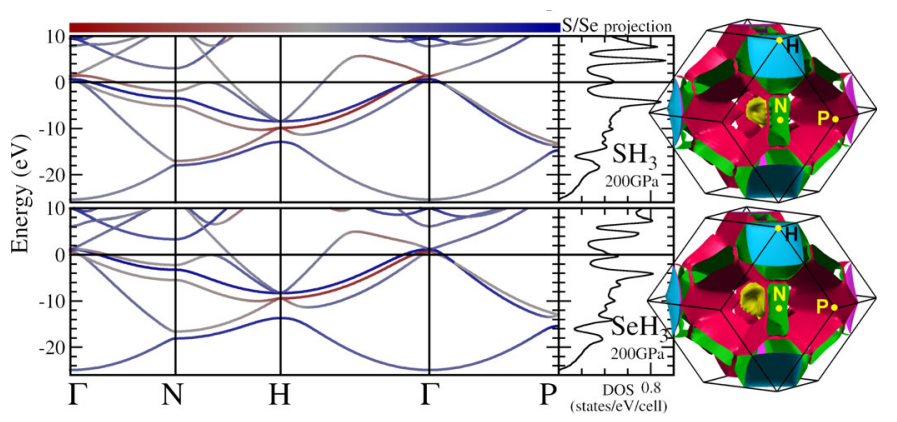

Fig. 3. Fermi surfaces and electronic band structures of $\mathrm{H}_{3} \mathrm{~S}$ and $\mathrm{H}_{3} \mathrm{Se}$ at high pressure in the $\operatorname{Im} \overline{3} m$ phase. The color-scale in the band lines indicates the projection of the KS states on the atomic orbitals of the sulfur/selenium atom normalized by the maximum total atomic projection of these valence states that is of about $70 \%$.

type), therefore depending on experimental conditions this rhombohedral phase may occur as a metastable one. The sequence of transformation is highlighted in Figure 1 by means of shaded areas. Different low-lying enthalpy structures found for $\mathrm{H}_{2} \mathrm{Se}, \mathrm{H}_{3} \mathrm{Se}$, and $\mathrm{H}_{4} \mathrm{Se}$ compositions are shown in Figure 2. Structural details are given in Supplemental Material ${ }^{\star}$. It is worth mentioning that we found $15 \mathrm{GPa}$ shift in the pressure at which occurs the sequence off phase transformations if one compares the LDA or GGA enthalpies for $\mathrm{H}_{x} \mathrm{~S}$. On the other hand, a much marked dependence (avobe $15 \mathrm{GPa}$ ) is found in the sequence of structure transformations in $\mathrm{H}_{x}$ Se. The main difference for H-Se is that in GGA-PBE the elemental decomposition becomes favourable at low pressures $(<150)$. However, both functionals lead to the same result at high pressure limit.

We focus now on the properties of $\mathrm{H}_{3} \mathrm{~S}$ and $\mathrm{H}_{3} \mathrm{Se}$ in the pressure range of stability of the $\operatorname{Im} \overline{3} m$ structure. The two materials show very similar properties. At $200 \mathrm{GPa}$ electronic band dispersions and Fermi surfaces are barely distinguishable, as seen in Figure 3. And in the range of pressure between 100 to $200 \mathrm{GPa}$ there are no significant changes in the electronic properties apart from the overall bandwidth that increases with pressure. An important aspect of the electronic structure is the presence of several Fermi surface sheets, with no marked nesting features and with Fermi states both at low and high momentum vector. At small momentum (close to the $\Gamma$-point, center of the Brillouin zone in Fig. 3) there are three small Fermi surfaces (only the green larger one can be seen in the figure, smaller ones being inside it). However, these provide a rather small contribution to the total density of states (DOS) at the Fermi level which mostly comes from the two larger Fermi surface sheets. These are of hybrid character, meaning that their Kohn-Sham (KS) states overlap both with $\mathrm{H}$ and $\mathrm{S} /$ Se states (the overlap is expressed in the figure by the color-scale of the band lines), suggesting that they will be coupled with both hydrogen and S/Se lattice vibrations (more details on this point will be given below). Overall the DOS shows a square root behaviour of the $3 \mathrm{D}$ electron gas, the main deviation from this occurs 
Table 1. Calculated critical temperatures and gaps. $\lambda$ is the electron phonon coupling parameter $[68,69] ; \omega_{\text {log }}$ is the logarithmic average of the $\alpha^{2} F$ function $[68,69] ; T_{\mathrm{c}}^{\mathrm{SCDFT}}$ is the critical temperature from SCDFT including RPA screened Coulomb repulsion; $T_{\mathrm{c}}^{\mathrm{SCDFT}, \mathrm{ph}}$ is the phonon only SCDFT critical temperature; $T_{\mathrm{c}}^{\mathrm{AD}, \mu^{*}=0}$ is the critical temperature from the Allen-Dynes modified McMillan formula [69-71], at $\mu^{*}=0$ (i.e. with no Coulomb pairing); $T_{\mathrm{c}}^{\mathrm{AD}, \mu^{*}=0.1}$ is the same $\mathrm{AD}$ formula but with the conventional value of $\mu^{*}=0.1 ; \Delta(T=0)$ is the superconducting gap computed [72] from the SCDFT calculations.

\begin{tabular}{ccccccccc}
\hline & & $\lambda$ & $\omega_{\log }$ & $T_{\mathrm{c}}^{\text {SCDFT }}$ & $\Delta(T=0)$ & $T_{\mathrm{c}}^{\text {SCDFT,ph }}$ & $T_{\mathrm{c}}^{\mathrm{AD}, \mu^{*}=0.1}$ & $T_{\mathrm{c}}^{\mathrm{AD}, \mu^{*}=0}$ \\
\hline $\mathrm{H}_{3} \mathrm{~S}$ & $200 \mathrm{GPa}$ & 2.41 & $109 \mathrm{meV}$ & $180 \mathrm{~K}$ & $43.8 \mathrm{meV}$ & $284 \mathrm{~K}$ & $255 \mathrm{~K}$ & $338 \mathrm{~K}$ \\
$\mathrm{D}_{3} \mathrm{~S}$ & $200 \mathrm{GPa}$ & 2.41 & $82 \mathrm{meV}$ & $141 \mathrm{~K}$ & $32.9 \mathrm{meV}$ & $216 \mathrm{~K}$ & $188 \mathrm{~K}$ & $247 \mathrm{~K}$ \\
$\mathrm{H}_{3} \mathrm{~S}$ & $180 \mathrm{GPa}$ & 2.57 & $101 \mathrm{meV}$ & $195 \mathrm{~K}$ & $44.8 \mathrm{meV}$ & $297 \mathrm{~K}$ & $250 \mathrm{~K}$ & $331 \mathrm{~K}$ \\
\hline $\mathrm{H}_{3} \mathrm{Se}$ & $200 \mathrm{GPa}$ & 1.45 & $120 \mathrm{meV}$ & $131 \mathrm{~K}$ & $28.4 \mathrm{meV}$ & $234 \mathrm{~K}$ & $174 \mathrm{~K}$ & $246 \mathrm{~K}$ \\
$\mathrm{H}_{3} \mathrm{Se}$ & $150 \mathrm{GPa}$ & 1.38 & $107 \mathrm{meV}$ & $110 \mathrm{~K}$ & $23.4 \mathrm{meV}$ & $195 \mathrm{~K}$ & $145 \mathrm{~K}$ & $209 \mathrm{~K}$ \\
$\mathrm{H}_{3} \mathrm{Se}$ & $100 \mathrm{GPa}$ & 1.76 & $87 \mathrm{meV}$ & $123 \mathrm{~K}$ & $27.0 \mathrm{meV}$ & $198 \mathrm{~K}$ & $156 \mathrm{~K}$ & $214 \mathrm{~K}$ \\
\hline
\end{tabular}

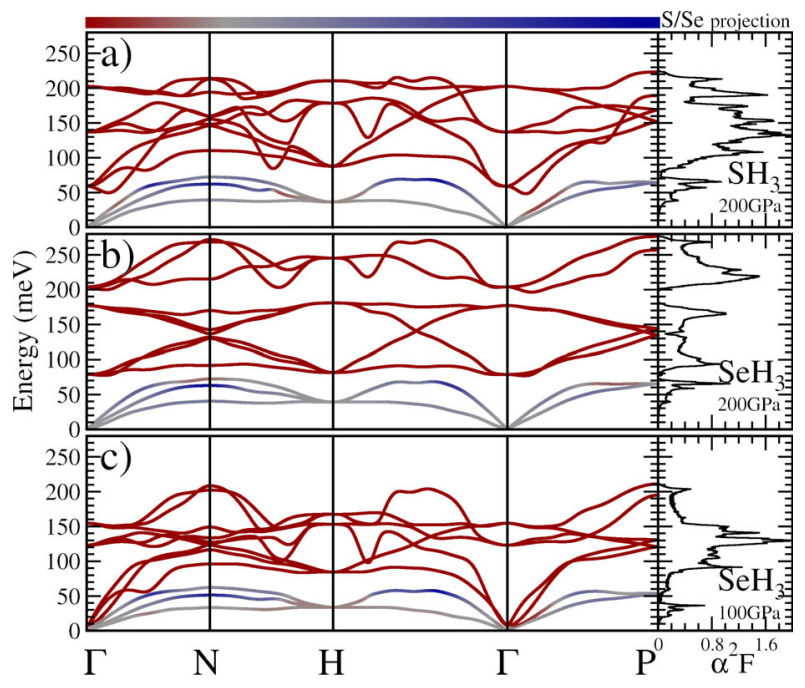

Fig. 4. Phonon dispersion and $\alpha^{2} F$ functions $[68,69]$ of $\mathrm{H}_{3} \mathrm{~S}$ and $\mathrm{H}_{3} \mathrm{Se}$ at high pressure in the cubic $\operatorname{Im} \overline{3} m$ structure. The color coding gives the projection of the mode displacement on the S/Se atom.

close to the Fermi energy where a peak with an energy width of about $2 \mathrm{eV}$ is present.

Unlike the electronic structure, phonons are strongly pressure and material dependent. Clearly a key role is played by the occurrence of the II order $R 3 m$ to $\operatorname{Im} \overline{3} m$ phase transition. This means that one of the high-energy stretching mode (originally above $200 \mathrm{meV}$ ) slowly softens at $\Gamma$. This softening can be clearly seen in $\mathrm{H}_{3} \mathrm{~S}$ at $200 \mathrm{GPa}$ (Fig. 4a) where a H-S stretching mode went down to about $60 \mathrm{meV}$. Eventually, as pressure lowers this softens to zero energy, marking the occurrence of the phase transition, at about $150 \mathrm{GPa}$ in $\mathrm{H}_{3} \mathrm{~S}$ and slightly below $100 \mathrm{GPa}$ in $\mathrm{H}_{3} \mathrm{Se}$. In fact, at $100 \mathrm{GPa}$ this mode has, in $\mathrm{H}_{3} \mathrm{Se}$, almost zero energy (see Fig. 4c).

In spite of these important changes in the phononic energy dispersion, the overall coupling strength $[68,69] \lambda$ does not show large variations over the pressure range, as we can see from Table 1. Naturally the coupling increases near the phase transition due the optical mode softening, however, as this is restricted to a relatively small region near the $\Gamma$ point, the effect is not dramatic. On the other hand there is definitively a difference in the coupling strength of the Se $(\lambda \sim 1.5)$ with respect to the $\mathrm{S}$ system $(\lambda \sim 2.5)$, indicating that selenium, due to its larger ionic size, provides a better electronic screening of the hydrogen vibrations.

We have computed, by means of SCDFT, critical temperatures of $\mathrm{H}_{3} \mathrm{~S}$ and $\mathrm{H}_{3} \mathrm{Se}$ in the pressure range of stability of the $\operatorname{Im} \overline{3} m$ structure, these are collected in Table 1. The predicted $T_{\mathrm{C}}$ for the $\mathrm{H}_{3} \mathrm{~S}$ system is $180 \mathrm{~K}$ at $200 \mathrm{GPa}$ and $195 \mathrm{~K}$ at $180 \mathrm{GPa}$, in agreement with the measured value of $185 \mathrm{~K}$ at $177 \mathrm{GPa}$. On the other hand our prediction for the deuterium substituted system $\mathrm{D}_{3} \mathrm{~S}$ is $141 \mathrm{~K}$, at $200 \mathrm{GPa}$. That is much larger than the measured [41] $T_{\mathrm{C}}$ of $90 \mathrm{~K}$. This huge experimental isotope effect (i.e. the dependence of the critical temperature on the nuclear mass) is therefore not consistent with our calculations. However the good agreement obtained with the $T_{\mathrm{C}}$ of the $\mathrm{H}_{3} \mathrm{~S}$ system seem to exclude an explanation in terms of anharmonic effects in the hydrogen vibrations, as suggested in reference [73] and confirmed in reference [37]. Nevertheless, the theoretical isotope coefficients $\alpha^{S}=0.05$ and $\alpha^{H}=0.4$ (defined as $\alpha^{A}=-\frac{M^{A}}{T c} \frac{\partial T_{C}}{\partial M^{A}}$, and computed at $200 \mathrm{GPa}$ with a three point numerical differentiation) clearly indicate and confirm [73] the dominant contribution of hydrogen phonon modes to the superconducting pairing.

Our prediction for $\mathrm{H}_{3} \mathrm{Se}$ at $200 \mathrm{GPa}$ is of $131 \mathrm{~K}$, this reduction of $T_{\mathrm{C}}$ is clearly due to the substitution of $\mathrm{S}$ and not an isotope effect. As mentioned before, it is caused instead, by a different coupling strength of the hydrogen modes in the Se environment. In spite of the lowered coupling strength $\lambda$ the reduction of $T_{\mathrm{C}}$ is not very large with respect to the sulfur system, as expected from the fact that the critical temperature at high coupling increases with the square root of $\lambda$ (while it is exponential at low coupling) $[68,69]$.

There are two aspects of these systems that are uncommon and make the use of conventional ${ }^{1}$ Eliashberg methods difficult to apply, while they are naturally handled in SCDFT. First, the strong variation of the electronic density of states at the Fermi level, that is pinned to a rather sharp peak in the DOS, second the extremely large el-ph

\footnotetext{
${ }^{1}$ Conventional implementation of the Eliashberg equations due to their computational cost, usually assume a $k$-independent pairing and a flat density of states. Anisotropic implementations [74] are not intrinsically affected by this limit.
} 
coupling and phonon frequencies that lead to a very broad region around $E_{\mathrm{F}}$ where the interaction is dominated by phonons over Coulomb repulsion.

The effect of the energy dependence of the DOS can be seen by comparing Eliashberg results with SCDFT when neglecting the Coulomb interaction (see Tab. 1). At $200 \mathrm{GPa}$ the two theories ${ }^{2}$ disagree by $54 \mathrm{~K}$, SCDFT giving $284 \mathrm{~K}$ while Eliashberg gives $338 \mathrm{~K}$. This difference comes from the energy dependence of the DOS, while Eliashberg assumes a flat DOS, in the SCDFT we can easily check this assumption by assuming a flat DOS, and for this case the SCDFT calculation would lead to $334 \mathrm{~K}$, in agreement with the Eliashberg result. Overall the critical temperature results to be quite sensitive to the shape of the DOS at the Fermi level and on how it is computed.

Physically the reduction of $T_{\mathrm{C}}$, occurring when the real DOS is considered, arises from the fact that the phononic pairing extends in a rather large region around the Fermi level, over the DOS peak structure of these systems (see Fig. 3). Beyond the range of the phononic pairing the coupling is dominated by the Coulomb interaction. As, in the static limit, this is repulsive, a superconducting system compensates it by a phase shift in the gap (i.e. in the quasi-particle orbitals), therefore making this repulsion contribute to the condensation (in unconventional superconductors exactly the same happens but directly at the Fermi level). This mechanism is called Coulomb renormalization $[69,75]$. The phase shift occurs at $\left|\epsilon_{\mathbf{k}}\right| \gtrsim \omega_{\log }$ but the scattering processes become less and less important as $\left|\epsilon_{\mathbf{k}}\right|$ increases (going down as $1 / \epsilon$ ). Therefore the most important energy region is where the DOS of the $\mathrm{H}_{3} \mathrm{~S}$ and $\mathrm{H}_{3}$ Se systems shows a dip, implying that the phase space available for this process is small and its effect weak. Note that in order to reproduce the $T_{\mathrm{C}}$ coming from SCDFT within the Allen-Dynes (AD) formula one should assume a $\mu^{*}$ of 0.16 , that is actually much larger than the value of $\mu$ itself $(\simeq 0.1)$.

The superconducting pairing is distributed over many phonon modes and over the Brillouin zone in q-space, despite the presence of several Fermi surface sheets and with different orbital character across the Fermi level, we obtained an isotropic (weakly k-dependent) gap at the Fermi level and the effect of anisotropy [76] on $T_{\mathrm{C}}$ is negligible $(<1 \mathrm{~K})$.

In summary, we have presented a fully ab-initio theoretical description on the crystal structure and superconductivity of $\mathrm{H}_{3} \mathrm{~S}$. An extensive structural search confirms the $\mathrm{H}_{3} \mathrm{~S}$ stoichiometry as the most stable configuration at high pressure. Our results confirm $\mathrm{H}_{3} \mathrm{~S}$ to be the material

\footnotetext{
${ }^{2}$ We are actually not reporting the Eliashberg result but that coming from the Allen-Dynes (AD) formula. The reason for this choice is that the two approaches agree perfectly (the difference being less than $1 \mathrm{~K}$ ) for the phonon case. But in addition when including $\mu^{*}$ the $\mathrm{AD}$ formula depends only on it, while the Eliashberg equations also depend on the Coulomb frequency cut-off (that changes the meaning of the $*$ in $\mu^{*}$ ). If we want to use a conventional value of $\mu^{*}$ between 0.1 and 0.15 [69] it is then better to use the parametrized $A D$ version of the Eliashberg method.
}

with the highest known superconducting critical temperature. The mechanism of superconductivity is clearly the same that was predicted for metallic hydrogen $[4,6,8]$ : the combined effect of high characteristic frequency due to hydrogen light mass and strong coupling due to the lack of electronic core in hydrogen. Still the working pressures of this superconductor are too high for any technological application [77]. Nevertheless the discovery of metallic superconducting hydrogenic bands already at $150 \mathrm{GPa}$ gives hope that further theoretical and experimental research in this direction may lead to even lower hydrogen metallization pressures and higher temperatures of superconductivity. Here we predict that $\mathrm{H}_{3} \mathrm{Se}$ is stable in the cubic $\operatorname{Im} \overline{3} m$ structure already at $100 \mathrm{GPa}$ with a very high $T_{\mathrm{C}}$ of $123 \mathrm{~K}$, a value which is comparable to cuprate superconductors.

J.A.F.L. acknowledges the financial support from EU's 7th Framework Marie-Curie Program within the "ExMaMa" Project (329386).

\section{References}

1. K. Shimizu, K. Amaya, N. Suzuki, J. Phys. Soc. Jpn 74, $1345(2005)$

2. C. Buzea, K. Robbie, Supercond. Sci. Technol. 18, R1 (2005)

3. E. Wigner, H.B. Huntington, J. Chem. Phys. 3, 764 (1935)

4. N. Ashcroft, Phys. Rev. Lett. 21, 1748 (1968)

5. C.F. Richardson, N.W. Ashcroft, Phys. Rev. Lett. 78, 118 (1997)

6. P. Cudazzo, G. Profeta, A. Sanna, A. Floris, A. Continenza, S. Massidda, E. Gross, Phys. Rev. Lett. 100, 257001 (2008)

7. L. Zhang, Y. Niu, Q. Li, T. Cui, Y. Wang, Y. Ma, Z. He, G. Zou, Solid State Commun. 141, 610 (2007)

8. P. Cudazzo, G. Profeta, A. Sanna, A. Floris, A. Continenza, S. Massidda, E.K.U. Gross, Phys. Rev. B 81, 134505 (2010)

9. J. Bardeen, L.N. Cooper, J.R. Schrieffer, Phys. Rev. 108, 1175 (1957)

10. C.J. Pickard, R.J. Needs, Nat. Phys. 3, 473 (2007)

11. P. Cudazzo, G. Profeta, A. Sanna, A. Floris, A. Continenza, S. Massidda, E. Gross, Phys. Rev. B 81, $134506(2010)$

12. P. Loubeyre, F. Occelli, R. LeToullec, Nature 416, 13 (2002)

13. M.I. Eremets, I.A. Troyan, Nat. Mater. 10, 927 (2011)

14. C.S. Zha, Z. Liu, R. Hemley, Phys. Rev. Lett. 108, 146402 (2012)

15. I.I. Naumov, R.J. Hemley, Accounts Chem. Res. 47, 3551 (2014)

16. N. Ashcroft, Phys. Rev. Lett. 92, 187002 (2004)

17. M.I. Eremets, I.A. Trojan, S.A. Medvedev, J.S. Tse, Y. Yao, Science 319, 1506 (2008)

18. X. Chen, V.V. Struzhkin, Y. Song, A.F. Goncharov, M. Ahart, Z. Liu, H.k. Mao, R.J. Hemley, Proc. Natl. Acad. Sci. 105, 20 (2008)

19. O. Degtyareva, J.E. Proctor, C.L. Guillaume, E. Gregoryanz, M. Hanfland, Solid State Commun. 149, 1583 (2009) 
20. M. Hanfland, J.E. Proctor, C.L. Guillaume, O. Degtyareva, E. Gregoryanz, Phys. Rev. Lett. 106, 095503 (2011)

21. T. Strobel, P. Ganesh, M. Somayazulu, P. Kent, R. Hemley, Phys. Rev. Lett. 107, 255503 (2011)

22. J.S. Tse, Y. Yao, K. Tanaka, Phy. Rev. Lett. 98, 117004 (2007)

23. X.J. Chen, V.V. Struzhkin, Y. Song, A.F. Goncharov, M. Ahart, Z. Liu, H.k. Mao, R.J. Hemley, Proc. Natl. Acad. Sci. 105, 20 (2008)

24. D.Y. Kim, R.H. Scheicher, S. Lebègue, J. Prasongkit, B. Arnaud, M. Alouani, R. Ahuja, Proc. Natl. Acad. Sci. 105, 16454 (2008)

25. J. Feng, R.G. Hennig, N.W. Ashcroft, R. Hoffmann, Nature 451, 445 (2008)

26. S. Wang, H.K. Mao, X.J. Chen, W.L. Mao, Proc. Natl. Acad. Sci. 106, 14763 (2009)

27. Y. Yao, D.D. Klug, Proc. Natl. Acad. Sci. 107, 20893 (2010)

28. G. Gao et al., Proc. Natl. Acad. Sci. 107, 1317 (2010)

29. D.Y. Kim, R.H. Scheicher, H.K. Mao, T.W. Kang, R. Ahuja, Proc. Natl. Acad. Sci. 107, 2793 (2010)

30. Y. Li, G. Gao, Y. Xie, Y. Ma, T. Cui, G. Zou, Proc. Natl. Acad. Sci. 107, 15708 (2010)

31. D. Zhou, X. Jin, X. Meng, G. Bao, Y. Ma, B. Liu, T. Cui, Phys. Rev. B 86, 014118 (2012)

32. Y. Li, J. Hao, H. Liu, Y. Li, Y. Ma, J. Chem. Phys. 140, $174712(2014)$

33. Y. Wang, Y. Ma, J. Chem. Phys. 140, 040901 (2014)

34. J. Hooper, T. Terpstra, A. Shamp, E. Zurek, J. Phys. Chem. C 118, 6433 (2014)

35. D. Duan, Y. Liu, F. Tian, D. Li, X. Huang, Z. Zhao, H. Yu, B. Liu, W. Tian, T. Cui, Sci. Rep. 4, 6968 (2014)

36. N. Bernstein, C.S. Hellberg, M.D. Johannes, I.I. Mazin, M.J. Mehl, Phys. Rev. B 91, 060511 (2015)

37. I. Errea, M. Calandra, C.J. Pickard, J. Nelson, R.J. Needs, Y. Li, H. Liu, Y. Zhang, Y. Ma, F. Mauri, Phys. Rev. Lett. 114, $157004(2015)$

38. D. Duan, X. Huang, F. Tian, D. Li, H. Yu, Y. Liu, Y. Ma, B. Liu, T. Cui, Phys. Rev. B 91, 180502 (2015)

39. R. Akashi, M. Kawamura, S. Tsuneyuki, Y. Nomura, R. Arita, Phys. Rev. B 91, 224513 (2015)

40. J.A. Flores-Livas, M. Amsler, T.J. Lenosky, L. Lehtovaara, S. Botti, M.A.L. Marques, S. Goedecker, Phys. Rev. Lett. 108, 117004 (2012)

41. A.P. Drozdov, M.I. Eremets, I.A. Troyan, arXiv : 1412.0460 [cond-mat.supr-con] (2014)

42. A.P. Drozdov, M.I. Eremets, I.A. Troyan, V. Ksenofontov, S.I. Shylin, Nature 525, 73 (2015)

43. E.J. Nicol, J.P. Carbotte, Phys. Rev. B 91, 220507 (2015)

44. A. Bianconi, T. Jarlborg, Europhys. Lett. 112, 37001 (2015)

45. D.A. Papaconstantopoulos, B.M. Klein, M.J. Mehl, W.E. Pickett, Phys. Rev. B 91, 184511 (2015)

46. C. Heil, L. Boeri, Phys. Rev. B 92, 060508 (2015)

47. J.A. Flores-Livas, A. Sanna, E.K.U. Gross, arXiv: $1501.06336(2015)$

48. S. Goedecker, J. Chem. Phys. 120, 9911 (2004)

49. S. Goedecker, W. Hellmann, T. Lenosky, Phys. Rev. Lett. 95, $055501(2005)$
50. M. Amsler, S. Goedecker, J. Chem. Phys. 133, 224104 (2010)

51. L.N. Oliveira, E.K.U. Gross, W. Kohn, Phys. Rev. Lett. 60, 2430 (1988)

52. M. Amsler et al., Phys. Rev. Lett. 108, 065501 (2012)

53. M. Amsler, J.A. Flores-Livas, T.D. Huan, S. Botti, M.A.L. Marques, S. Goedecker, Phys. Rev. Lett. 108, 205505 (2012)

54. S. Botti, J.A. Flores-Livas, M. Amsler, S. Goedecker, M.A.L. Marques, Phys. Rev. B 86, 121204 (2012)

55. E. Bitzek, P. Koskinen, F. Gähler, M. Moseler, P. Gumbsch, Phys. Rev. Lett. 97, 170201 (2006)

56. G. Kresse, J. Furthmüller, Comput. Mat. Sci. 6, 15 (1996)

57. X. Gonze et al., Comput. Phys. Commun. 180, 2582 (2009)

58. P. Giannozzi et al., J. Phys.: Condens. Matter 21, 395502 (2009)

59. M. Lüders, M.A.L. Marques, N.N. Lathiotakis, A. Floris, G. Profeta, L. Fast, A. Continenza, S. Massidda, E.K.U. Gross, Phys. Rev. B 72, 024545 (2005)

60. M.A.L. Marques, M. Lüders, N.N. Lathiotakis, G. Profeta, A. Floris, L. Fast, A. Continenza, E.K.U. Gross, S. Massidda, Phys. Rev. B 72, 024546 (2005)

61. A. Floris, A. Sanna, S. Massidda, E.K.U. Gross, Phys. Rev. B 75, 054508 (2007)

62. R.S. Gonnelli et al., Phys. Rev. Lett. 100, 207004 (2008)

63. A. Sanna, G. Profeta, A. Floris, A. Marini, E.K.U. Gross, S. Massidda, Phys. Rev. B 75, 020511 (2007)

64. G. Profeta, C. Franchini, N. Lathiotakis, A. Floris, A. Sanna, M.A.L. Marques, M. Lüders, S. Massidda, E.K.U. Gross, A. Continenza, Phys. Rev. Lett. 96, 047003 (2006)

65. J.A. Flores-Livas, A. Sanna, Phys. Rev. B 91, 054508 (2015)

66. F. Essenberger, A. Sanna, A. Linscheid, F. Tandetzky, G. Profeta, P. Cudazzo, E.K.U. Gross, Phys. Rev. B 90, 214504 (2014)

67. M. Einaga, M. Sakata, T. Ishikawa, K. Shimizu, M. Eremets, A. Drozdov, I. Troyan, N. Hirao, Y. Ohishi, arXiv: $1509.03156(2015)$

68. J.P. Carbotte, Rev. Mod. Phys. 62, 1027 (1990)

69. P.B. Allen, B. Mitrović, Theory of Superconducting Tc, in Solid State Physics (Academic Press, 1983), Vol. 37

70. P.B. Allen, R.C. Dynes, Phys. Rev. B 12, 905 (1975)

71. W.L. McMillan, Phys. Rev. 167, 331 (1968)

72. A. Sanna, E.K.U. Gross (2016), to be published

73. D.A. Papaconstantopoulos, B.M. Klein, M.J. Mehl, W.E. Pickett, Phys. Rev. B 91, 184511 (2015)

74. E.R. Margine, F. Giustino, Phys. Rev. B 87, 024505 (2013)

75. P. Morel, P.W. Anderson, Phys. Rev. 125, 1263 (1962)

76. H. Suhl, B. Matthias, L. Walker, Phys. Rev. Lett. 3, 552 (1959)

77. P.F. McMillan, Nat. Mater. 1, 1476 (2002)

Open Access This is an open access article distributed under the terms of the Creative Commons Attribution License (http://creativecommons.org/licenses/by/4.0), which permits unrestricted use, distribution, and reproduction in any medium, provided the original work is properly cited. 